EPJ manuscript No.

(will be inserted by the editor)

\title{
The Detector and Interaction Region for a Photon Collider at TESLA
}

\author{
Aura Rosca \\ DESY Zeuthen, Platanenallee 6, 15738 Zeuthen, Germany
}

Received: October 3, 2003

\begin{abstract}
TESLA is designed as an electron-positron linear collider (LC) based on super-conducting technology. A second interaction region is forseen to be incorporated in the design allowing its possible operation as a photon collider. In this paper I describe the basic design of the $\gamma \gamma$ interaction region taking into account the beam-beam and laser related issuses and review some aspects of other accelerator components such as the feedback system and the beam dump which are critical to the operation of TESLA as a photon collider.
\end{abstract}

PACS. XX.XX.XX No PACS code given

\section{Introduction}

The collisions of high energy photon beams will provide new opportunities for particle physics, ranging from particle searches to the measurement of the Higgs boson properties.

The cross sections for charged pair particle production in $\gamma \gamma$ collisions are about one order of magnitude higher than in $\mathrm{e}^{+} \mathrm{e}^{-}$collisions [1. Therefore, one can study new particles far from threshold with higher rate. For example, WW pair production at $500 \mathrm{GeV}$ is a factor of 10 larger than in $\mathrm{e}^{+} \mathrm{e}^{-}$. About $2 \cdot 10^{6} \mathrm{WW}$ pairs could be produced at a photon collider with a luminosity of $100 \mathrm{fb}^{-1}$ thus allowing a precise study of the anomalous gauge boson interactions. Also, the cross sections for the production of charged scalar, lepton and top pairs are a factor of 5 to 10 higher at a photon collider than at an $\mathrm{e}^{+} \mathrm{e}^{-} \mathrm{LC}[1$.

But the main motivation for the construction of a photon collider is to measure the properties of the Higgs bosons. A photon collider permits a direct measurement of the two photon decay width of the Higgs boson with a precision better than 2\% 2] for a Higgs boson with a mass of $120 \mathrm{GeV}$. The coupling of the Higgs to two photons proceeds through loops to which any charged particle that gets its mass through the Higgs mechanism will contribute, no matter how heavy it is. A measurement of the $\gamma \gamma$ width is thus very sensitive to new physics. At a photon collider the Higgs bosons are produced on resonance by the fusion of two photons. This means a higher discovery reach for the heavier Higgs bosons. Very important is the determination of the CP nature of the Higgs bosons which could be done at a photon collider using linearly polarized photon beams.

The beams for a photon collider can be obtained by converting the high energy electron beams at a LC into

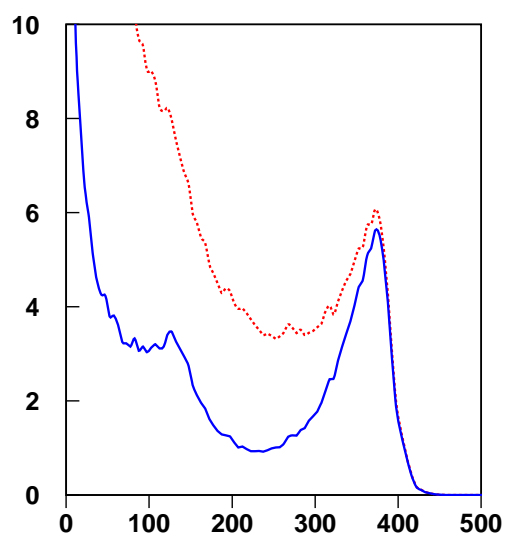

Fig. 1. $\gamma \gamma$ luminosity spectra at TESLA. The solid line shows the luminosity distribution for total helicity of the photons 0 and the dotted line for the case the total helicity 2 is also included.

high energy photons through Compton backscattering [3] of high density laser photons with an energy of approximative $1 \mathrm{eV}$, a few millimeter before the interaction point. The high energy photons will follow the direction of motion of the electrons and will collide at the interaction point.

The maximum energy of the generated photons is given by $\mathrm{E}_{\gamma}^{\max }=x E_{\mathrm{e}} /(1+x)$, with $E_{\mathrm{e}}$ being the electron beam energy and $x=\frac{4 \omega_{\mathrm{L}} E_{\mathrm{e}}}{m_{\mathrm{e}}^{2} c^{4}}$, where $\omega_{\mathrm{L}}, m_{\mathrm{e}}$ and $c$ are the laser photon energy, the electron rest mass, and the velocity of light, respectively. It can reach up to $80 \%$ of the initial electron beam energy. 
Table 1. The laser requirements

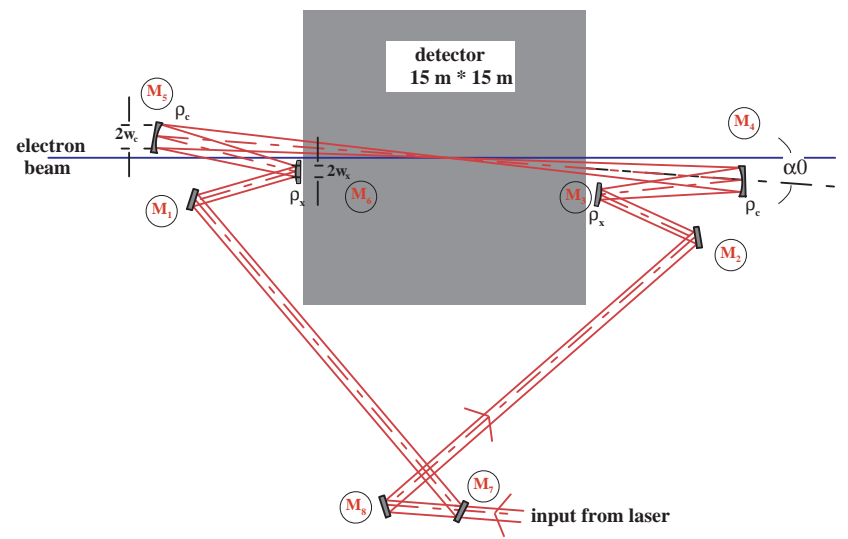

Fig. 2. A schematic view of the laser cavity proposed for the TESLA photon collider.

The luminosity distribution in $\gamma \gamma$ collisions has a high energy peak, see Figure 1 which is the most useful for the measurements, and a low energy part. The luminosity in the hard part of the spectrum can be up to $10 \%$ [4 of the geometric luminosity of the electron beam.

The Compton scattered electrons will have a large energy spread and will undergo a significant disruption during the beam-beam interaction. To ensure a clean extraction of these electrons, the photon collider requires a crossing angle at the interaction point, to allow a larger pipe for the outgoing beam. The current TESLA design has a crab crossing angle at the $\gamma \gamma$ interaction point of $34 \mathrm{mrad}$ 4.

To accomodate the extraction lines with larger apertures, as well as the laser beams in the interaction region, the design of this region for $\mathrm{e}^{+} \mathrm{e}^{-}$collisions must be modified for a $\gamma \gamma$ collider. In addition, also a mask system which protects the vertex detector from the background caused by beam-beam interactions should be installed here.

In the remaining of this paper several technical issues of the photon collider will be discussed, together with some open items which still require studies before the construction of such a collider becomes feasible.

\section{The laser and optical systems}

The laser system must match the bunch structure of the electron beam, which is 2800 bunches in a train with 5 trains per second for TESLA, and to provide the required laser photon density at the conversion point in order to convert efficiently the electrons into high energy photons. For efficient conversion of $250 \mathrm{GeV}$ electrons, the optimal laser wavelength is about $1 \mu \mathrm{m}$ and the pulse energy is 5 $\mathrm{J}$, with a pulse duration of about 1 ps. The laser requirements for the TESLA photon collider are summarized in Table 1

\begin{tabular}{ll}
\hline Parameter & Requirement \\
\hline Laser wavelength & $\lambda \approx 1 \mu \mathrm{m}$ \\
Laser energy & $E_{\text {pulse }} \approx 5 \mathrm{~J}$ \\
Pulse duration & $\tau \approx 1-3 \mathrm{ps}$ \\
Rayleigh length & $Z_{\mathrm{r}} \approx 0.4 \mathrm{~mm}$ \\
Repetition rate & TESLA collision rate \\
Average power & $P \approx 70 \mathrm{~kW}$ \\
\hline
\end{tabular}

Due to the long pulse separation and the large number of pulses per train at TESLA, the required average power of the laser system is very high and cannot be generated at present. It has been proposed to increase the laser power by means of an optical resonant cavity [5] with a quality factor reaching 100. The length of the cavity has to be such that the round trip of the laser pulses inside matches the bunch separation of the electron bunches in the train, and has to be stabilized within $0.5 \mathrm{~nm}$. This results in about $100 \mathrm{~m}$ cavity length at TESLA. Figure 2 shows a drawing of the optical cavity consisting of several mirrors, all mounted around the detector. The laser radiation is transfered into the cavity through a coupling mirror and brought to the interaction region with two final focusing mirrors placed away from the beam pipe. The crossing angle between the laser pulse and the electron beam is determined by how small the focusing mirrors can be realised without producing significant losses of the laser pulses circulating inside the cavity. The laser collision angle reduces the conversion probability which can be compensated by a higher laser energy. A value of about $60 \mathrm{mrad}$ [6] is forseen in the present design.

Laser pulses with an energy of several Joules and a duration of the order of picoseconds can be achieved with modern laser technology. The wavelength of the most powerful solid-state lasers is about $1 \mu \mathrm{m}$, which is exactly the required value for the TESLA photon collider.

\section{Backgrounds and the detector design}

A problem at the photon collider are the high backgrounds, which can be a factor of 10 or higher than at the LC. Plotted in Figure 3] is the energy distribution from the beam-beam interactions at the distance of $3.8 \mathrm{~m}$ after the interaction point. The circle shows the size of the beam pipe of the outgoing beam. The magnetic field sweeps a significant energy out of the beam pipe, which amounts to more than $1 \mathrm{TeV} / \mathrm{mm}^{2}$.

Figure 4 shows the masking system design to reduce the background in the central tracking detectors. The whole region of the outgoing beams is shielded by two conical tungsten masks. The outer mask is extended up to the vertex detector. The maximum number of hits in the layers of the vertex from the incoherent pairs is about 372 hits, which corresponds to a hit density of $0.03 \mathrm{hits} / \mathrm{mm}^{2}$ on the first layer. This hit density is tolerable for the current design of the vertex detector. The number of photons 


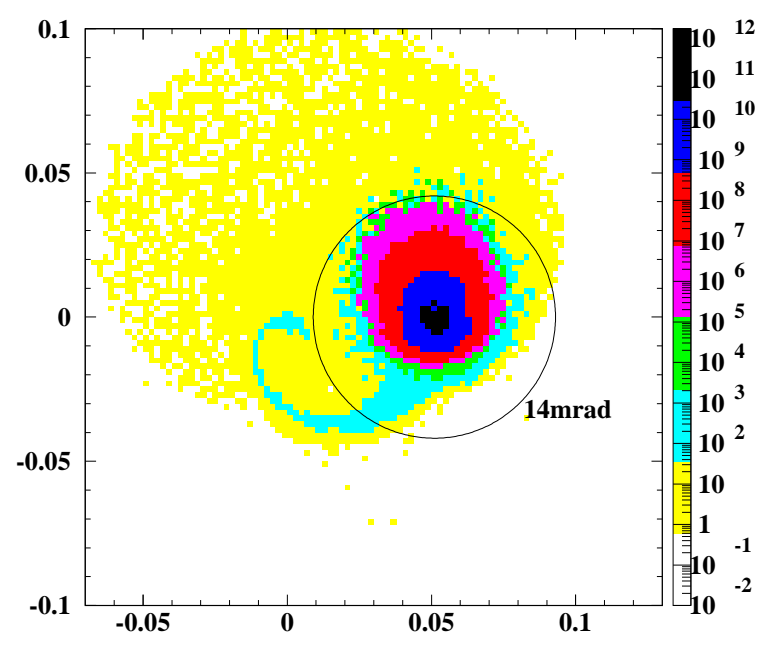

Fig. 3. The energy distribution on the calorimeter face at a distance of $3.8 \mathrm{~m}$ away from the interaction point.

entering the TPC is about a factor 2.4 larger than in the $\mathrm{e}^{+} \mathrm{e}^{-}$case, leading to an occupancy below $1 \%$, which can be handled in the track reconstruction. The detectors outside the forward region can be a copy of the LC detector design.

\section{Open issues: collision stability and the beam dump}

Beam steering needs to be performed at two positions: at the photon-photon interaction point to keep in collision beams with a size of $88 \mathrm{~nm} \times 4.3 \mathrm{~nm}$, and at the two Compton interaction points, with an expected laser beam size of $14 \mu \mathrm{m} \times 14 \mu \mathrm{m}$. Details are still to be worked out and several ideas are investigated.

At the $\gamma \gamma$ interaction point, first the electron beams are stabilized by a fast feedback system which measures the deflection of the beams, using undisrupted electron bunches at the beginning of the train. The steering of the photon beams relies on the fact that they follow the electron direction of motion and that the electron beams are stable over the entire train. Only after the electron beams are brought into collision the laser will be turned on. For the Compton interaction points, it would be possible to separate electrons and positrons from the photons, and with an instrumented beam dump to measure the amount of high energy photons in the beam, which is an indication of a good alignment of the laser.

The beam dump is an open subject: the problem is that the photon beam cannot be deflected by electromagnetic fields, and this has two drawbacks. There is a direct line of sight from the interaction point to the dump, which is a problem due to the neutrons traveling back to the detector. Also, the photon beam will always hit the same spot on the window, causing a high thermal load and high radiation damage to the window.

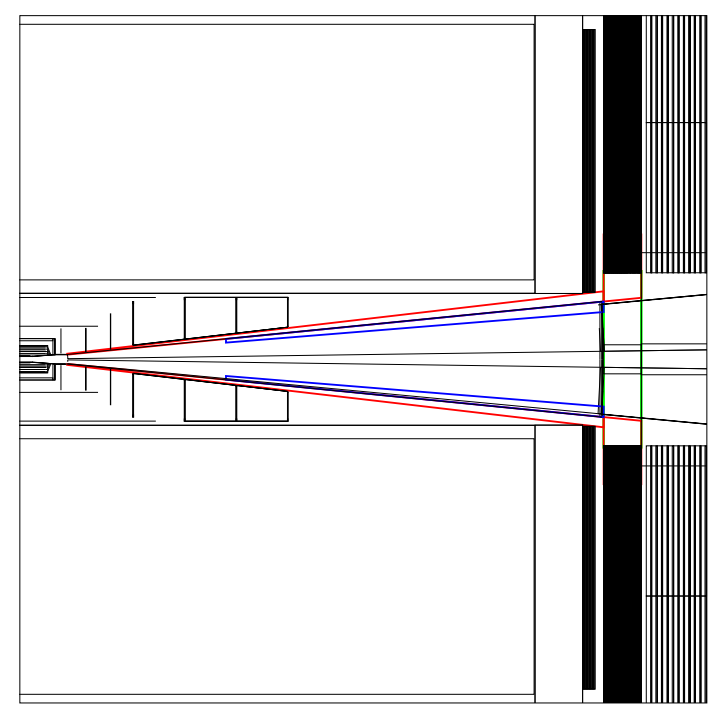

Fig. 4. A schematic view of the mask system to protect the tracking detectors against the beam background.

In conclusion, although this is a work in progress, there is no fundamental obstacle which would stop TESLA to operate as a photon collider.

\section{Acknoledgements}

I would like to thank F. Bechtel, G. Klemz, K. Mönig, J. Sekaric and A. Stahl for help with the preparation of this presentation.

\section{References}

1. V. Telnov, Nucl. Inst. and Meth. A355 (1995) 3.

2. G. Jikia and S. Söldner-Rembold, Nucl. Inst. and Meth. A472 (2001) 133; P. Niezurawski, A.F. Zarnecki and M. Krawczyk, Acta Physica Polonica B34 (2003) 177; P. Niezurawski, A.F. Zarnecki and M. Krawczyk, hep-ph/0307183 A. Rosca and K. Mönig, LC-PHIM-2003-084, hep-ph/0310036

3. I.F. Ginzburg et al., JETP Lett. 34 (1982) 491; I.F. Ginzburg et al., Nucl. Inst. Meth. 205 (1983) 47; I.F. Ginzburg et al., Nucl. Inst. Meth. A219 (1984) 5.

4. TESLA, Technical Design Report, vol. VI, Appendices.

5. I. Will et al., Nucl. Inst. Meth. A472 (2001) 79.

6. G. Klemz, private communication 